\section{Efficient Methods of Clutter Suppression for Coexisting Land and Weather Clutter Systems}

The removal of coexisting land and weather clutter signals is a common problem in many radar applications. Although the optimal method for land-weather clutter suppression is known, it is usually not amenable to implementation due to computation and storage limitations of the processor. We propose an alternative method that employs low rank approximations on weather clutter correlation matrix to obtain detectors which are feasible to implement. The performance of the proposed method is compared with the optimal detector and some other detectors used for this task to illustrate the trade-off between the improvement factor gain and the computational, storage requirements.

\section{INTRODUCTION}

We consider the problem of clutter cancellation for pulsed Doppler radar systems. The conventional clutter cancellation problem studies the cancellation of a single component clutter. This problem has been studied in depth and various methods have been proposed [1-4]. The problem of clutter cancellation when the clutter is due to two sources, such as coexisting land and weather systems, has not been explored in as much detail as the conventional problem. In this paper, we present a novel method for joint land-weather clutter cancellation and compare its performance with the optimal and some other alternative methods.

The problem of coexisting land and weather clutter frequently occurs in the over-the-horizon radar systems [5-8]. For such systems, the return from a desired target is contaminated by the returns from both a land system having zero Doppler frequency and a weather system having a non-zero Doppler frequency. In particular, the surveillance operation in rainy and foggy conditions can be severely compromised by the weather clutter unless measures are taken [9].

Manuscript received January 8, 2008; revised July 23 and October 27, 2008; released for publication October 29, 2008.

IEEE Log No. T-AES/45/4/935117.

Refereeing of this contribution was handled by F. Gini.

This work was partially supported by the Scientific and Technological Research Council of Turkey under Grant 106E187 and by the ASELSAN Corporation.

0018-9251/09/\$26.00 (C) 2009 IEEE
The land clutter cancellation problem is well studied in the literature. Moving target indicator (MTI) and Doppler processing techniques with discrete Fourier transform (DFT) banks or optimized filterbanks have been studied in depth along with the optimal clutter autocovariance-based approaches $[2,3]$. On the other hand, there has been less elaboration of weather clutter cancellation. In [10], a simple MTI operation, whose null is at the Doppler frequency of weather clutter, is suggested to suppress the weather clutter. In some applications, a window function is utilized to suppress the leakage of weather clutter with the center Doppler frequency $f_{w}$ to the neighboring Doppler frequencies. In [11] a comparison is given to illustrate the performance of the mentioned MTI-based systems for weather clutter cancellation with the optimal canceller. In [11] it has been noted that the clutter suppression performance of MTI-based systems can be sufficient under some scenarios and but in general a filter bank with optimized filters is required for an acceptable operation. A difficulty in the optimization of the moving target detector (MTD) filters is the large number of degrees of freedom such as weather clutter power level, its central Doppler frequency, land clutter power and the distance between the Doppler frequency of interest (look Doppler) and the central frequency of the weather clutter.

Four generations of MTD filters have been developed. MTD-I uses simple Doppler processing. MTD-II and MTD-III can provide higher improvement factor gains but require a careful control on pulse train lengths to realize this gain $[12,13]$. MTD-IV has been used in airport surveillance radar systems [14]. MTD filters are specially designed narrowband filters with extremely low sidelobes to suppress clutter. MTD filters can be considered as ad-hoc solutions to the clutter suppression problem. The suitability of an MTD filter to a scenario requires the matching of actual clutter powers and the center Doppler frequency of the weather system to the design specifications. In [15] MTD filters that can be adjusted for three different levels of land clutter power with extremely low sidelobes have been constructed. Their performance has been examined in [11]. MTD filters using time-frequency distributions have been proposed [16]. Recently MTD filters using fractional correlation [17] and using neural networks have been suggested [18]. A brief history of MTD filters written by one of the pioneers of the field can be found in [19].

In this paper a suboptimal method performing sufficiently close to the optimal solution is developed with a realizable computational complexity. Different from MTD filters, the proposed method does not require a filter design approach but it is based on an approximation to the optimal method. Therefore the proposed method does not require a 
scenario-dependent optimization procedure for the calculation of filter coefficients. The proposed method is in principle similar to the rank-reduction techniques applied in space-time adaptive processing [20]. Here we use rank-reduction techniques to achieve an operating point in the trade-off between performance, computational complexity, and storage requirements.

The paper is organized as follows. In Section II we describe the problem, the clutter models, and the optimal solution. In Section III we describe the MTI approach, whitening approach, and the proposed approximations to the optimal method. In Section IV we present numerical comparisons under various operational scenarios after which the paper is concluded.

\section{PROBLEM DESCRIPTION}

A train of $N$ pulses are used to detect the presence of a target in a range cell. The received vector $\mathbf{r}$ is assumed to contain the I/Q samples of the signal represented with the complex baseband representation:

$$
\mathbf{r}=\alpha \mathbf{s}+\sqrt{E_{l}} \mathbf{c}_{l}+\sqrt{E_{w}} \mathbf{c}_{w}+\sqrt{N_{o}} \mathbf{n} .
$$

In (1), the vector $\mathbf{s}$ is the desired signal denoting the return from the target and the vectors $\mathbf{c}_{l}$ and $\mathbf{c}_{w}$ are statistically independent clutter return vectors due to land and weather systems. The vector $\mathbf{n}$ represents the effect of thermal noise. The signal $\mathbf{s}$ is the Doppler steering vector where its coefficient $\alpha$ is a complex random variable. The clutter terms are assumed to be the samples of wide-sense stationary process whose autocorrelation is given below. The entries of $\mathbf{n}$ are independent identically distributed, circularly symmetric, complex Gaussian random variables with zero mean and unit variance. The parameters $\alpha, E_{l}, E_{w}, N_{o}$ are scalars to represent the power of each term.

The vector $\mathbf{s}$ is assumed to be a deterministic quantity in the form $\left[1 \gamma \gamma^{2} \ldots \gamma^{N-1}\right]$ where $\gamma=$ $e^{-j 2 \pi f_{t} / \mathrm{PRF}}$ (PRF is pulse-repetition frequency). The parameter $f_{t}$ corresponds to the Doppler frequency of the target (in Hertz). The goal is to detect the deterministic signal, which is the complex exponential vector, in the presence of random disturbance due to clutter and thermal noise.

As in [10] and [11], the autocorrelation of land clutter and weather clutter vectors $\mathbf{c}_{l}, \mathbf{c}_{w}$ are assumed to be in the form

$$
\mathbf{r}_{c}(k)=\rho^{k^{2}} e^{-j 2 \pi f_{w} k / \mathrm{PRF}} .
$$

In (2), $f_{w}$ is the Doppler frequency (in Hertz) of the clutter object. For land clutter $f_{w}$ is equal to zero, whereas $f_{w}$ is proportional to the radial velocity of the weather clutter. In this paper, we continue with the assumption of [10] and [11] that the weather and land clutter have the same type of autocorrelation function but with different Doppler frequencies.

Under these conditions, the input signal-to-interference and noise ratio (SINR) for each received sample becomes $\operatorname{SINR}^{\text {in }}=|\alpha|^{2} /\left(E_{l}+E_{c}+\right.$ $N_{o}$ ). The goal of MTI or Doppler processing is to increase the SINR by jointly processing $N$ samples of vector $\mathbf{r}$.

A linear combination of the received samples can be expressed as $\mathbf{w}^{\mathrm{H}} \mathbf{r}$, where $\mathbf{w}$ is a linear combination vector of length $N$. After linear combination, SINR at the output becomes

$$
\operatorname{SINR}^{\text {out }}=|\alpha|^{2} \frac{\mathbf{w}^{\mathrm{H}} \mathbf{S s}^{\mathrm{H}} \mathbf{w}}{\mathbf{w}^{\mathrm{H}} \mathbf{R} \mathbf{w}}
$$

where the matrix $\mathbf{R}$ denotes the autocorrelation matrix of the clutter and thermal noise terms. More explicitly, the $i$ th row and $k$ th column of the matrix $\mathbf{R}$ is

$$
\mathbf{R}(i, k)=E_{l} r_{l}(i-k)+E_{w} r_{w}(i-k)+N_{o} \delta(i-k)
$$

where the terms $r_{l}$ and $r_{w}$ refer to land and weather clutter autocorrelations whose definitions are given in (2).

When $\mathbf{s}$ is a deterministic parameter, all the components of SINR ${ }^{\text {out }}$ in (3) are deterministic, and therefore the vector $\mathbf{w}$ maximizing the ratio in (3) is the generalized eigenvector of matrix $\mathbf{R}$ and rank-1 matrix $\mathbf{s s}^{\mathrm{H}}$. The optimal vector is given by $\mathbf{w}_{\text {opt }}=\mathbf{R}^{-1} \mathbf{s}$, [21]. The optimal weight vector can be written as follows:

$$
\begin{aligned}
\mathbf{w}_{\mathrm{opt}} & =\mathbf{R}^{-1} \mathbf{s} \\
& =(E_{l} \mathbf{R}_{l}+\underbrace{E_{w} \mathbf{D}_{f_{w}} \mathbf{R}_{l} \mathbf{D}_{f_{w}}^{\mathrm{H}}}_{\mathbf{R}_{w}}+N_{o} \mathbf{I})^{-1} \mathbf{s} \\
& =\frac{1}{N_{o}}\left(\frac{E_{l}}{N_{o}} \mathbf{R}_{l}+\frac{E_{w}}{N_{o}} \mathbf{D}_{f_{w}} \mathbf{R}_{l} \mathbf{D}_{f_{w}}^{\mathrm{H}}+\mathbf{I}\right)^{-1} \mathbf{s} .
\end{aligned}
$$

In (5), the matrix $\mathbf{R}_{l}$ denotes the normalized autocorrelation matrix for land clutter. The weather clutter matrix $\mathbf{R}_{w}$ is represented in terms of $\mathbf{R}_{l}$ through prior and posterior multiplication by $\mathbf{D}_{f_{w}}$ after (2). The matrix $\mathbf{D}_{f_{w}}$ is a diagonal matrix with entries $\left\{1, \gamma, \gamma^{2}, \ldots, \gamma^{N-1}\right\}$ and $\gamma=e^{-j 2 \pi f_{w} / \mathrm{PRF}}$.

We note that the land clutter correlation matrix and weather clutter correlation matrix are similarity transformations of each other. Therefore both matrices have the same eigenvalues [22]. We denote the eigendecomposition of the land clutter correlation matrix as $\mathbf{R}_{l}=\mathbf{E} \boldsymbol{\Lambda} \mathbf{E}^{\mathrm{H}}$ where $\mathbf{E}$ is the matrix whose columns are composed of the eigenvectors of $\mathbf{R}_{l}$ and $\Lambda$ is a diagonal matrix with associated eigenvalues on the diagonal. Using the eigendecomposition in (5), one can find

$$
\mathbf{w}_{\mathrm{opt}}=\frac{1}{N_{o}}\left(\frac{E_{l}}{N_{o}} \mathbf{E} \boldsymbol{\Lambda} \mathbf{E}^{\mathrm{H}}+\frac{E_{w}}{N_{o}}\left(\mathbf{D}_{f_{w}} \mathbf{E}\right) \boldsymbol{\Lambda}\left(\mathbf{D}_{f_{w}} \mathbf{E}\right)^{\mathrm{H}}+\mathbf{I}\right)^{-1} \mathbf{s} .
$$


From the last equation, it can be noted that when one of the clutter terms is dominant, then the processor simplifies. When weather clutter is weak in comparison with the combination of land clutter and noise, the optimal filter becomes

$$
\mathbf{w}_{\mathrm{opt}}^{l}=\frac{1}{N_{o}}\left(\frac{E_{l}}{N_{o}} \mathbf{E} \boldsymbol{\Lambda} \mathbf{E}^{\mathrm{H}}+\mathbf{I}\right)^{-1} \mathbf{s} .
$$

On the contrary, when land clutter is negligible, the following optimal weight vector is found as follows by recalling $\mathbf{D}_{f_{w}} \mathbf{D}_{f_{w}}^{\mathrm{H}}=\mathbf{I}$

$$
\begin{aligned}
\mathbf{w}_{\mathrm{opt}}^{w} & =\frac{1}{N_{o}}\left(\frac{E_{w}}{N_{o}} \mathbf{D}_{f_{w}} \mathbf{E} \boldsymbol{\Lambda} \mathbf{E}^{\mathrm{H}} \mathbf{D}_{f_{w}}^{\mathrm{H}}+\mathbf{D}_{f_{w}} \mathbf{D}_{f_{w}}^{\mathrm{H}}\right)^{-1} \mathbf{s} \\
& =\frac{1}{N_{o}}\left(\mathbf{D}_{f_{w}}\left[\frac{E_{w}}{N_{o}} \mathbf{E} \boldsymbol{\Lambda} \mathbf{E}^{\mathrm{H}}+\mathbf{I}\right] \mathbf{D}_{f_{w}}^{\mathrm{H}}\right)^{-1} \mathbf{s} \\
& =\frac{1}{N_{o}} \mathbf{D}_{f_{w}}\left[\frac{E_{w}}{N_{o}} \mathbf{E} \boldsymbol{\Lambda} \mathbf{E}^{\mathrm{H}}+\mathbf{I}\right]^{-1} \underbrace{\mathbf{D}_{f_{w}}^{\mathrm{H}} \mathbf{s}}_{\hat{\mathbf{s}}} .
\end{aligned}
$$

In the equation above, $\hat{s}$ denotes look Doppler vector after the frequency shift by $-f_{w}$. For example, if look Doppler vector is at $f_{l}=0.75 \times \mathrm{PRF}$ and $f_{w}$ is at $0.5 \times \mathrm{PRF}$, then $\hat{s}=\mathbf{D}_{f_{w}}^{\mathrm{H}} \mathbf{s}$ is equivalent to a look Doppler vector at frequency $0.25 \times$ PRF. With this interpretation, it can be noted that the optimal filter to cancel the weather clutter in the absence of land clutter is the modulated (frequency shifted) version of the optimal land clutter cancellation filter.

We note that the matrix inversion operation in the optimal filter has to be calculated for every range cell. In many land clutter suppression systems, the clutter power in a cell is estimated during the operation and stored in a clutter map. To use a clutter map, the clutter power and electronic noise level should be periodically estimated. The coefficients of the optimal filter $\left(\mathbf{w}_{\text {opt }}\right)$ is retreived from a look-up table using the estimated clutter power level as the table index. The storage for the look-up table is a critical resource in many applications. The land clutter removal system whose equation is given in (7) has only one free parameter $E_{l} / N_{o}$. Therefore a look-up table with a single index (dimension) is sufficient to store the coefficients. A filter strictly removing weather clutter (when the land clutter is absent) can be easily adapted from the stored land clutter removal filter coefficients using (8). But for a general system capable of removing coexisting land and weather clutter systems, the dimensions of the storage changes from 1 to 3, that is in addition to $E_{l} / N_{o}$ we need to store $E_{w} / N_{o}$ and $f_{w}$. Since the dimensions for the storage unit is tripled, the system may easily become infeasible to implement via precalculated look-up tables.

The central Doppler frequency of the weather clutter system is expected to show little variation across the range cells. The parameter $f_{w}$ can be estimated using the effected range cells in azimuth and range directions. In the literature a simple weighted averaging operation has been proposed as a viable solution [23]. In addition to this solution, one can also use other spectrum estimation techniques such as autoregressive modeling, pulse-pair processing, etc., for the same purpose [24].

In this paper, we present alternative methods for the solution of the problem with a feasible storage at the expense of some additional computation. We assume that the land clutter cancellation filters are precalculated and stored with $E_{l} / N_{o}$ parameter as the table index. In the following section, we present the proposed approximation to the optimal method in addition to two other alternatives.

\section{PROPOSED TECHNIQUES}

We present three methods for bimodal clutter removal in this section. The implementation complexity and storage requirements of these methods are also discussed.

\section{A. Shifted MTI Followed by Land-Only Filter}

As suggested in [10], the receive vector $\mathbf{r}$ is first processed with a single-order MTI cancellation filter whose null frequency is shifted to $f_{w}$ and then the land removal filter calculated from (7) is used to remove the effect of land clutter. The processing output of this system can be written as follows:

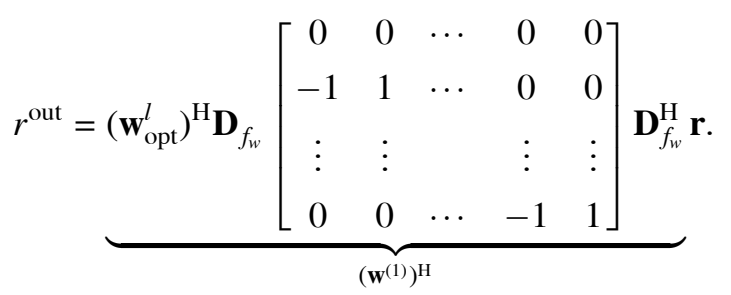

Discussion: The proposed system can be examined in two stages. In the first stage, the effect of weather clutter system is suppressed with the frequency-shifted MTI system. In the second stage, the land clutter is removed with the optimal land-only filters as if the weather clutter is completely removed after the first stage. Note that the shifted MTI operation takes into account neither the power of weather clutter signal nor the width of the clutter spectrum. As noted above, when the land clutter is the dominant term in comparison with the weather clutter, the optimal filter is close to the land clutter removal filter. Since a shifted MTI system applies the same filtering operation at all power levels, this system can neither adjust the depth of nulling or the width of the null depending on the application scenario. 
Computation and Storage Requirements: This system does not have any additional storage requirements for weather clutter suppression. It requires $2 N$ complex multiplications and $N-1$ complex additions per range cell in addition to the operation of land-clutter supression filter.

\section{B. Land-Only Filter Followed by Weather-Only Filter}

This system is composed of cascade filters for land clutter removal and weather clutter removal. For the proposed scheme, the land clutter is processed by $\mathbf{R}_{\ln }^{-1 / 2}$ matrix where $\mathbf{R}_{\ln }$ is the autocorrelation matrix of the land clutter and thermal noise $\mathbf{R}_{\mathrm{ln}}=$ $\left(E_{l} / N_{o}\right) \mathbf{R}_{l}+\mathbf{I}$. This operation corresponds to whitening of the signal when the weather clutter component is temporarily ignored. After this stage, the resultant vector is one more time processed with $\mathbf{R}_{\mathrm{wn}}^{-1 / 2}$ which is the whitening matrix for $\mathbf{r}$ in the absence of land clutter. Finally, the signal $\mathbf{s}$ is captured after these stages by matched filtering [25]. This method approximates the whitened matched filter solution which is known to be equivalent to the SINR maximizing solution given in (3). The approximate whitened matched filter vector becomes

$$
\mathbf{w}^{(2)}=\mathbf{R}_{\mathrm{ln}}^{-1 / 2} \mathbf{R}_{\mathrm{wn}}^{-1} \mathbf{R}_{\mathrm{ln}}^{-1 / 2} \mathbf{s} .
$$

Discussion: This system assumes that the autocorrelation matrix for land and weather system which is $\mathbf{R}=N_{o}\left(\left(E_{l} / N_{o}\right) \mathbf{R}_{l}+\left(E_{w} / N_{o}\right) \mathbf{R}_{w}+\mathbf{I}\right)$ can be approximately factorized as follows $\mathbf{R} \approx$ $N_{o}\left(\left(E_{l} / N_{o}\right) \mathbf{R}_{l}+\mathbf{I}\right)\left(\left(E_{w} / N_{o}\right) \mathbf{R}_{w}+\mathbf{I}\right)$. The accuracy of the approximation depends of the relative size of the cross term appearing in the right-hand side but missing on the left-hand side of the same relation. If $E_{l} E_{w} / N_{o}^{2}$ is small in comparison with the other components, the approximation is accurate. This method tries to suppress both sources of clutter in an ad-hoc manner based on the idea of sequentially whitening the spectrum.

Computation and Storage Requirements: This processor can be implemented by storing the inverses of both $\mathbf{R}_{\mathrm{ln}}^{1 / 2}$ and $\mathbf{R}_{\mathrm{wn}}$ matrices. The parameter space for two matrices is decoupled, that is $\left\{E_{l} / N_{o}\right\}$ for $\mathbf{R}_{\mathrm{ln}}^{-1 / 2}$ and $\left\{E_{w} / N_{o}, f_{w}\right\}$ for $\mathbf{R}_{\mathrm{wn}}^{-1}$. Therefore the storage requirements double in size but not in dimension. However, the increase in the computational complexity is quite significant since matrix multiplications are involved. The overall complexity is $3 N^{2}$ multiplications and $3 N(N-1)$ additions per range cell.

A second alternative for implementation with less storage requirements is the following. The matrix $\mathbf{R}_{w}$ can be factorized as follows: $\mathbf{R}_{\mathrm{wn}}=\mathbf{D}_{f_{w}} \mathbf{R}_{\mathrm{ln}} \mathbf{D}_{f_{w}}^{\mathrm{H}}$. The inverse of matrix $\mathbf{R}_{\mathrm{wn}}$ can then be calculated using the following relation: $\mathbf{R}_{\mathrm{wn}}^{-1}=\mathbf{D}_{f_{\mathrm{w}}} \mathbf{R}_{\mathrm{ln}}^{-1 / 2} \mathbf{R}_{\mathrm{ln}}^{-1 / 2} \mathbf{D}_{f_{\mathrm{w}}}^{\mathrm{H}}$. Therefore one can avoid the storage of $\mathbf{R}_{\mathrm{wn}}^{-1}$ matrices and can use stored $\mathbf{R}_{\mathrm{wn}}^{-1 / 2}$ matrices for its calculation.
The cost of not storing $\mathbf{R}_{\mathrm{wn}}^{-1}$ matrices is an additional computation of $2 N(N+1)$ multiplications and $2 N(N-1)$ additions per range cell.

A third implementation alternative can be increasing the look-up table dimension by one. The vector $\mathbf{w}^{(2)}$ in (10) can be stored to reduce number of operations. One should decide on one of these approaches based on the overall system resources.

\section{Proposed Method}

Equation (5) gives the optimal solution for arbitrary clutter powers and clutter Doppler frequency. An approximate yet computationally feasible approach is to approximate the weather clutter autocorrelation using lower rank matrices. Since the clutter matrix is highly correlated, very few terms can accurately represent the weather clutter matrix. The eigendecomposition of weather clutter is given as follows

$$
\mathbf{R}_{w}=\sum_{k=1}^{N} \lambda_{k} \mathbf{u}_{k} \mathbf{u}_{k}^{\mathrm{H}} .
$$

In (11), the vector $\mathbf{u}_{k}$ is the unit-norm eigenvector of $\mathbf{R}_{w}$ corresponding to the $k$ th largest eigenvalue $\lambda_{k}$. As stated in (8), the vectors $\mathbf{u}_{k}$ are the frequency-shifted versions of the eigenvectors of the land clutter matrix.

We propose to approximate $\mathbf{R}_{w}$ with rank-1 and rank-2 matrices and then apply the matrix inversion lemma to derive a Doppler processor for land-weather clutter system. Generalization to higher order approximations is possible but not pursued here to ease the presentation. The rank-1 and rank-2 approximation for $\mathbf{R}_{w}$ is given as follows

$$
\begin{aligned}
& \hat{\mathbf{R}}_{w}^{(1)}=\operatorname{tr}\left(\mathbf{R}_{w}\right) \mathbf{u}_{1} \mathbf{u}_{1}^{\mathrm{H}} \\
& \hat{\mathbf{R}}_{w}^{(2)}=\operatorname{tr}\left(\mathbf{R}_{w}\right)\left(\frac{\lambda_{1}}{\lambda_{1}+\lambda_{2}} \mathbf{u}_{1} \mathbf{u}_{1}^{\mathrm{H}}+\frac{\lambda_{2}}{\lambda_{1}+\lambda_{2}} \mathbf{u}_{2} \mathbf{u}_{2}^{\mathrm{H}}\right) .
\end{aligned}
$$

We note that the low rank approximations of $\mathbf{R}_{w}$ are scaled such that they have the same power with the full rank $\mathbf{R}_{w}$ matrix. That is, the approximations are weighted according to energy in each eigenvector direction and the total energy of the normalized clutter return vector (trace of $\mathbf{R}_{w}$ ) is matched with the approximations. We also note that $\operatorname{tr}\left(\mathbf{R}_{w}\right)=N$.

When the reduced rank approximations are substituted for $\mathbf{R}_{w}$ in (5) and the matrix inversion lemma is utilized, the following expressions are obtained:

$$
\begin{aligned}
\hat{\mathbf{w}}_{\mathrm{opt}}^{(1)} & =\frac{1}{N_{o}}\left(\frac{E_{l}}{N_{o}} \mathbf{R}_{l}+\frac{E_{w}}{N_{o}} \hat{\mathbf{R}}_{w}^{(1)}+\mathbf{I}\right)^{-1} \mathbf{s} \\
& =\frac{1}{N_{o}}\left(\mathbf{R}_{\ln }+\frac{E_{w}}{N_{o}} N \mathbf{u}_{1} \mathbf{u}_{1}^{\mathrm{H}}\right)^{-1} \mathbf{s} \\
& =\frac{1}{N_{o}}\left(\mathbf{R}_{\ln }^{-1}-\frac{1}{\sigma_{c}^{2}} \mathbf{c c}^{\mathrm{H}}\right) \mathbf{s}
\end{aligned}
$$


where $\mathbf{R}_{\ln }=E_{l} / N_{o} \mathbf{R}_{l}+\mathbf{I}, \mathbf{c}=\mathbf{R}_{\ln }^{-1} \mathbf{u}_{1}$, and $\sigma_{c}^{2}=$ $N_{o} /\left(N E_{w}\right)+\mathbf{u}_{1}^{\mathrm{H}} \mathbf{R}_{\ln }^{-1} \mathbf{u}_{1}$. Details on the application of the lemma are given in the appendix.

Discussion: Some important interpretations can be drawn from (14). With the weighting operation, the output of Doppler filter with the look direction $\mathbf{s}$ becomes $z(\mathbf{s})$

$$
z(\mathbf{s})=\frac{1}{N_{o}} \mathbf{s}^{\mathrm{H}} \mathbf{R}_{\ln }^{-1} \mathbf{r}-\frac{1}{\sigma_{c}^{2} N_{o}}\left(\mathbf{s}^{\mathrm{H}} \mathbf{R}_{\ln }^{-1} \mathbf{u}_{1}\right)\left(\mathbf{u}_{1}^{\mathrm{H}} \mathbf{R}_{\ln }^{-1} \mathbf{r}\right)
$$

The first term in (15) is the plain land clutter removal operation with projection onto the vector $\mathbf{R}_{\ln }^{-1} \mathbf{s}$ where the land clutter and thermal noise terms are whitened and matched filtered [25]. In the second term, the received vector is projected onto the weather clutter's strongest eigenvector in order to cancel it when obtaining the final result. The term $\mathbf{s}^{\mathrm{H}} \mathbf{R}_{\mathrm{ln}}^{-1} \mathbf{u}_{1}$ gives us the scalar used in the projection. The portion of the received signal in parallel with the weather clutter's strongest eigenvector is completely cancelled with this operation. In other words, the first term of $\hat{\mathbf{w}}_{\mathrm{opt}}^{(1)}$, which is $1 / N_{o} \mathbf{R}_{\ln }^{-1} \mathbf{s}$, stands for the cancellation of clutter in the absence of weather clutter. The second term is a correction on the first term when the weather clutter of power $E_{w}$ coexists. It can be noted that the effect of second terms vanishes as $E_{w} \rightarrow 0$ or $\sigma_{c}^{2} \rightarrow \infty$.

The proposed implementation explicitly makes use of the weather clutter power and its Doppler frequency in the selection of filter coefficients. We note that the suppression levels of two clutter sources are adjusted through the $\sigma_{c}^{2}$ parameter whose value is calculated through the matrix inversion lemma without any ad-hoc assumptions as has been done in previous methods. The success of the method solely depends on the accuracy of the low rank approximation.

When the same procedure is repeated for the rank-2 approximation, one can get the following

$$
\hat{\mathbf{w}}_{\mathrm{opt}}^{(2)}=\frac{1}{N_{o}}\left(\mathbf{R}_{\mathrm{ln}}^{-1}-C \mathbf{c c}^{\mathrm{H}}-D \mathbf{d d} \mathbf{d}^{\mathrm{H}}+E\left(\mathbf{d c}^{\mathrm{H}}+\mathbf{c d}^{\mathrm{H}}\right)\right) \mathbf{s} .
$$

The variables appearing in (16) are given as follows:

$$
\begin{aligned}
\mathbf{c} & =\mathbf{R}_{\mathrm{ln}}^{-1} \mathbf{u}_{1} \\
\mathbf{d} & =\mathbf{R}_{\mathrm{ln}}^{-1} \mathbf{u}_{2} \\
C & =1 / \sigma_{c}^{2}+|\gamma|^{2} /\left(\sigma_{c}^{4} \sigma_{d}^{2}\right) \\
D & =1 / \sigma_{d}^{2} \\
E & =2 \operatorname{Re}(\gamma) /\left(\sigma_{c}^{2} \sigma_{d}^{2}\right) .
\end{aligned}
$$

The parameters connecting the clutter power to the parameters used in (16) are

$$
\begin{aligned}
\gamma & =\mathbf{u}_{2}^{\mathrm{H}} \mathbf{R}_{\ln }^{-1} \mathbf{u}_{1} \\
\sigma_{c}^{2} & =N_{o} /\left(N E_{w}\right)\left(\lambda_{1}+\lambda_{2}\right) / \lambda_{1}+\mathbf{u}_{1}^{\mathrm{H}} \mathbf{R}_{\ln }^{-1} \mathbf{u}_{1} \\
\sigma_{d}^{2} & =N_{o} /\left(N E_{w}\right)\left(\lambda_{1}+\lambda_{2}\right) / \lambda_{2}+\mathbf{u}_{2}^{\mathrm{H}} \mathbf{R}_{\ln }^{-1} \mathbf{u}_{2}-|\gamma|^{2} / \sigma_{c}^{2} .
\end{aligned}
$$

Comments similar to those made for rank-1 approximation are also valid here. The first term in (16) is the land clutter removal filter. The following two terms cancel the components of the signal parallel to weather clutter's strongest eigenvectors. The very last term in (16) is related to $\left(\mathbf{s}^{\mathrm{H}} \mathbf{R}_{\ln }^{-1} \mathbf{u}_{1}\right)\left(\mathbf{u}_{2}^{\mathrm{H}} \mathbf{R}_{\ln }^{-1} \mathbf{r}\right)$. In the case that $\mathbf{R}_{\ln }^{-1} \mathbf{u}_{1}$ and $\mathbf{R}_{\ln }^{-1} \mathbf{u}_{2}$ projections are not orthogonal, there occurs an overcancellation after cancelling two terms. The last term, hence, is a correction term to remedy the overcancellation by the previous terms.

Computation and Storage Requirements: For the rank-1 approximation, there is no additional storage requirements for the proposed solution if the inverse of $\mathbf{R}_{\mathrm{ln}}$ matrices are stored in a look-up table. We note that $\mathbf{u}_{1}$ is a unit-norm vector with the description $\mathbf{u}_{1}=\mathbf{D}_{f_{w}} \mathbf{e}_{1}$, where $\mathbf{e}_{1}$ is the dominant eigenvector of $\mathbf{R}_{\mathrm{ln}}$. The vector $\mathbf{u}_{1}$ can be calculated online by a multiplication with a diagonal matrix, if $\mathbf{e}_{1}$ is precalculated and stored. The calculation of $\mathbf{u}_{1}$ requires $N$ multiplications and $N-1$ additions. The calculation of $\mathbf{c}$ and $\sigma_{c}^{2}$ requires a total of $N^{2}+N+1$ multiplications and $N^{2}$ additions. The implementation of $\left(1 / \sigma_{c}^{2}\right) \mathbf{s}^{\mathrm{H}} \mathbf{c c}^{\mathrm{H}} \mathbf{r}$ for the suppression of weather clutter further requires $2 N+1$ multiplications and $2 N-2$ additions. The overall cost of the weather clutter suppression is then $N^{2}+4 N+2$ multiplications and $N^{2}+3 N-3$ additions per range cell.

A second implementation can be proposed as follows: If the online calculation vector $\mathbf{c}$ is not feasible, the vectors $\mathbf{c}$ and the scalar $\mathbf{u}_{1}^{\mathrm{H}} \mathbf{R}_{\mathrm{ln}}^{-1} \mathbf{u}_{1}$ can be stored with the $f_{w}$ index. The parameter $\sigma_{c}^{2}$ can then be calculated on-line with 1 multiplications and 1 addition. This reduces the overall cost to $2 N+2$ multiplications and $2 N-1$ additions.

A third possible implementation is possible, if $\mathbf{R}_{\mathrm{ln}}^{-1} \mathbf{s}$ vectors for each $\mathbf{s}$ look Doppler direction of interest are stored instead of $\mathbf{R}_{\mathrm{ln}}^{-1}$ matrices. In a typical system, $N$ look vectors for $N$ Doppler bins are used. If we call these vectors as $\mathbf{s}_{k}$ vectors where $\mathbf{s}_{k}=\left[\begin{array}{lllll}1 & W & W^{2} & \ldots W^{N-1}\end{array}\right]$ and $W=e^{-i(2 \pi / N) k}$, one can then make use of the following relation

$$
\mathbf{u}_{1}^{\mathrm{H}} \mathbf{R}_{\mathrm{ln}}^{-1} \mathbf{r}=\left(\sum_{k=0}^{N-1} \alpha_{k} \mathbf{s}_{k}^{\mathrm{H}}\right) \mathbf{R}_{\mathrm{ln}}^{-1} \mathbf{r}
$$

to implement (15). This requires the storage of decomposition coefficients $\alpha_{k}$ for each value of weather clutter center frequency $f_{w}$. The computational cost of this alternative is the same as the second one.

The processor employing the rank-2 approximation has approximately 2 times the computational load for each of the implementation types discussed.

\section{NUMERICAL COMPARISONS}

In this section, we compare the performance of the methods presented in Section III. In Fig. 1, 


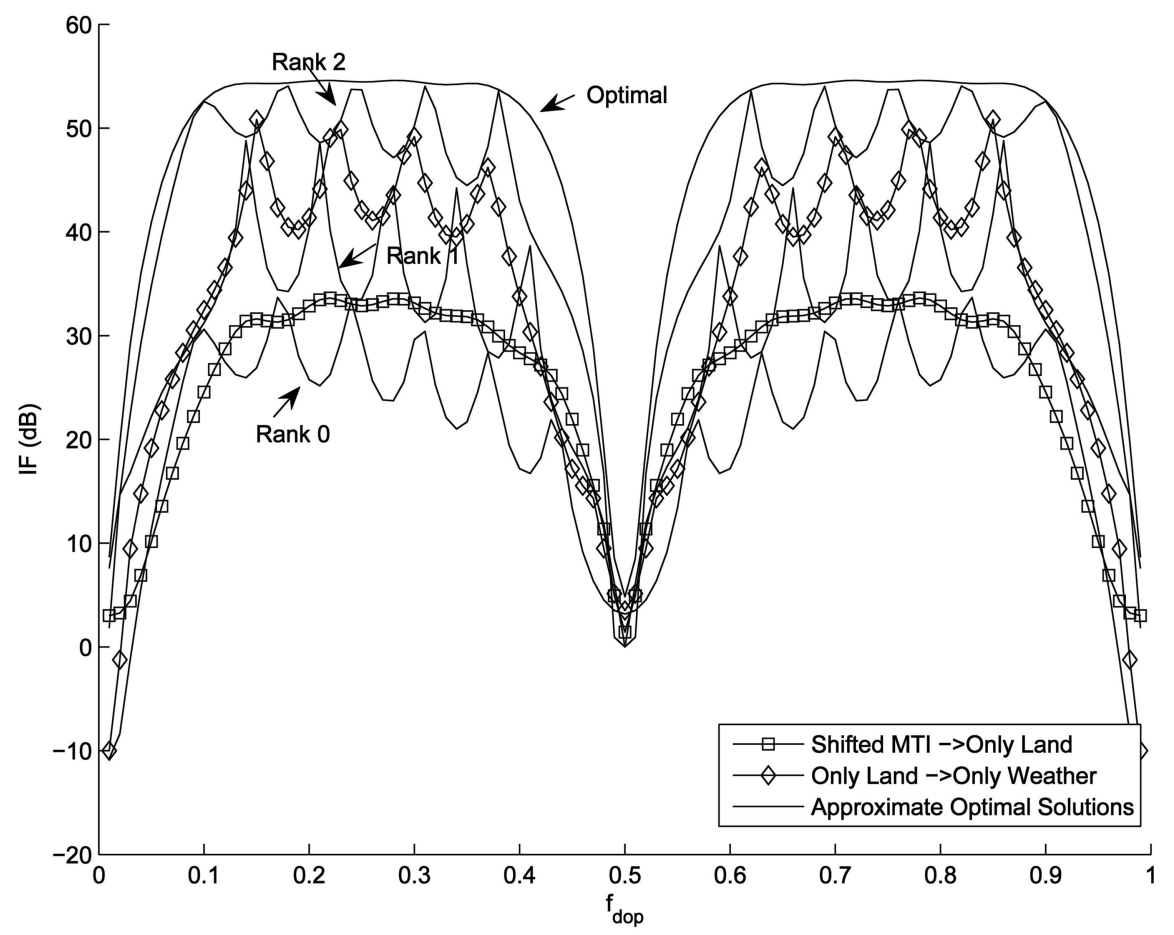

Fig. 1. $N=16, E_{l} / N_{o}=40 \mathrm{~dB}, E_{w} / N_{o}=40 \mathrm{~dB}, f_{w}=0.5$ PRF.

the improvement factor $\left(\mathrm{SINR}^{\text {out }} / \mathrm{SINR}^{\text {in }}\right.$ ) versus target Doppler frequency is shown. In this figure, the number of processing pulses is 16 , land and weather clutter both have $40 \mathrm{~dB}$ power above the thermal noise level, $\rho=0.999$, and the weather clutter has a Doppler frequency at 1/2 PRF. In Fig. 1, the improvement factor (IF) for the optimal solution and the proposed solutions are given. The solutions approximating the optimal solution are given for rank-0, rank- 1 , and rank- 2 cases. The rank- 0 case corresponds to ignoring the weather clutter completely and applying the land clutter filter as if weather clutter does not exist.

One can note from Fig. 1 that there is a significant loss of performance if the weather clutter is ignored. Shifted MTI results in some improvement and the sequential whitening method provides further improvements on MTI. The approximations to the optimal solution provide results close to the optimal solution for the rank-2 case.

In Fig. 2, the performance of the methods is compared when weather clutter has a Doppler frequency centered at $0.2 \mathrm{PRF}$. All other parameters are the same as the ones of Fig. 1. It can be noted that shifted MTI and the approximate whitening methods provide a similar performance under these conditions. The rank-2 approximation performs again close to the optimal solution.

In Fig. 3, the performance of the methods is compared when weather clutter power is $20 \mathrm{~dB}$ over $N_{o}$. All other parameters are the same as the ones of Fig. 2. It can be noted that shifted MTI presents a poorer performance than rank-0 approximation. A disadvantage of the shifted MTI method is that it cannot adapt to the power of weather clutter. In some scenarios such as presented in Fig. 3, ignoring the weather clutter can be more beneficial than applying the shifted MTI method. The second method which is the approximate whitening operation presents a performance close to optimal for this scenario. The rank-2 approximation is virtually identical to the optimal solution.

In [11], MTI and shifted MTI filters combination has been used to mitigate the land and weather clutter. To compare the performance of the proposed solution with the MTI-based approach, we have implemented MTI and shifted MTI with and without Hamming window at $E_{l} / N_{o}=60 \mathrm{~dB}, E_{w} / N_{o}=50 \mathrm{~dB}$, and $f_{w}=0.2$ PRF. The results are given in Fig. 4. As expected, the windowing operation leads to a poorer improvement factor around the clutter Doppler frequency. While for some other target Doppler frequencies, which are far away from the center of the clutter frequency, windowing significantly improves the performance of the MTI-based system. Even with the application of windowing, the performance of the MTI-based system has a significant performance gap from the optimal solution.

In Fig. 5, different from earlier figures, we present the frequency response of the MTI-based clutter suppression filters with and without Hamming window and the frequency response of the optimal filter. All filters are adjusted for the look Doppler of $0.5 \times$ PRF. As expected, the windowing operation 


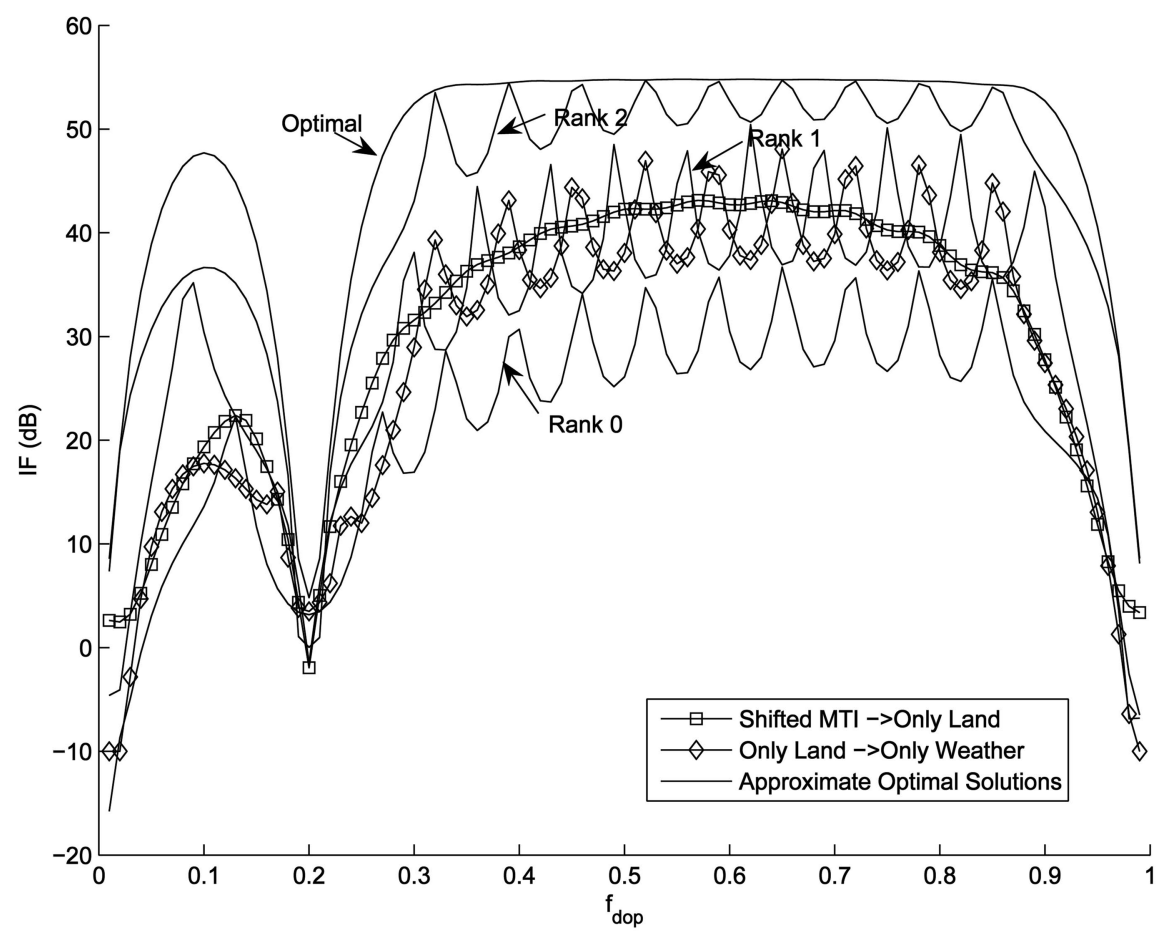

Fig. 2. $N=16, E_{l} / N_{o}=40 \mathrm{~dB}, E_{w} / N_{o}=40 \mathrm{~dB}, f_{w}=0.2 \mathrm{PRF}$.

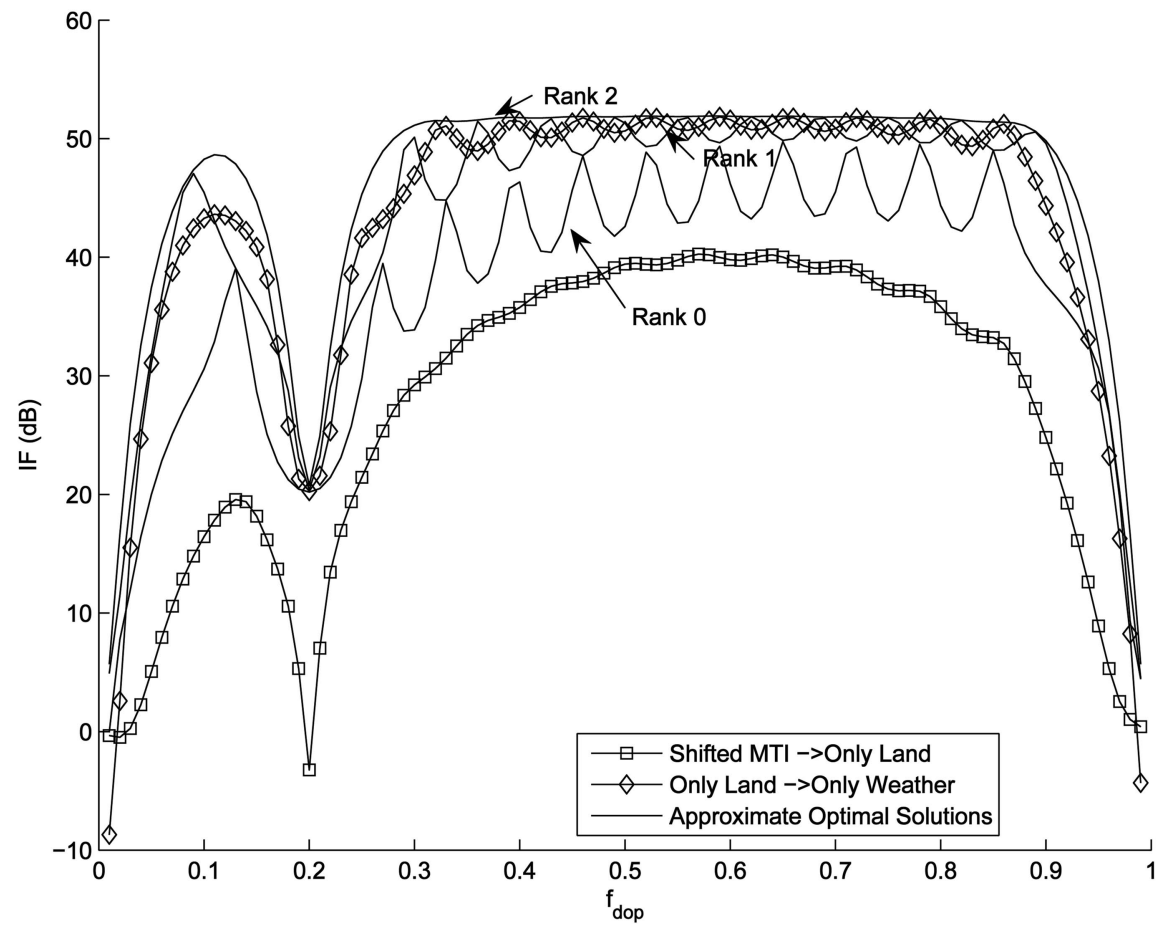

Fig. 3. $N=16, E_{l} / N_{o}=40 \mathrm{~dB}, E_{w} / N_{o}=20 \mathrm{~dB}, f_{w}=0.2$ PRF.

leads to the expansion of the mainlobe of the response, while the sidelobes are significantly suppressed. The optimal filter applies a $-90 \mathrm{~dB}$ power attenuation at the center of clutter Doppler frequency while the other filters apply $-30 \mathrm{~dB}$ and $-60 \mathrm{~dB}$ of attenuation. In addition, around the center frequency of the weather clutter, the windowed system produces much poorer results due to the expansion of the mainlobe of the filter. In general, due to the ad-hoc nature of MTI filters, the weather clutter cannot be much suppressed with MTI solutions.

From Figs. 4 and 5, we can say that MTI-based systems, while successfully applicable in some scenarios, can suffer from a poor performance when compared with the optimal solution. The approximations to the optimal system proposed in 


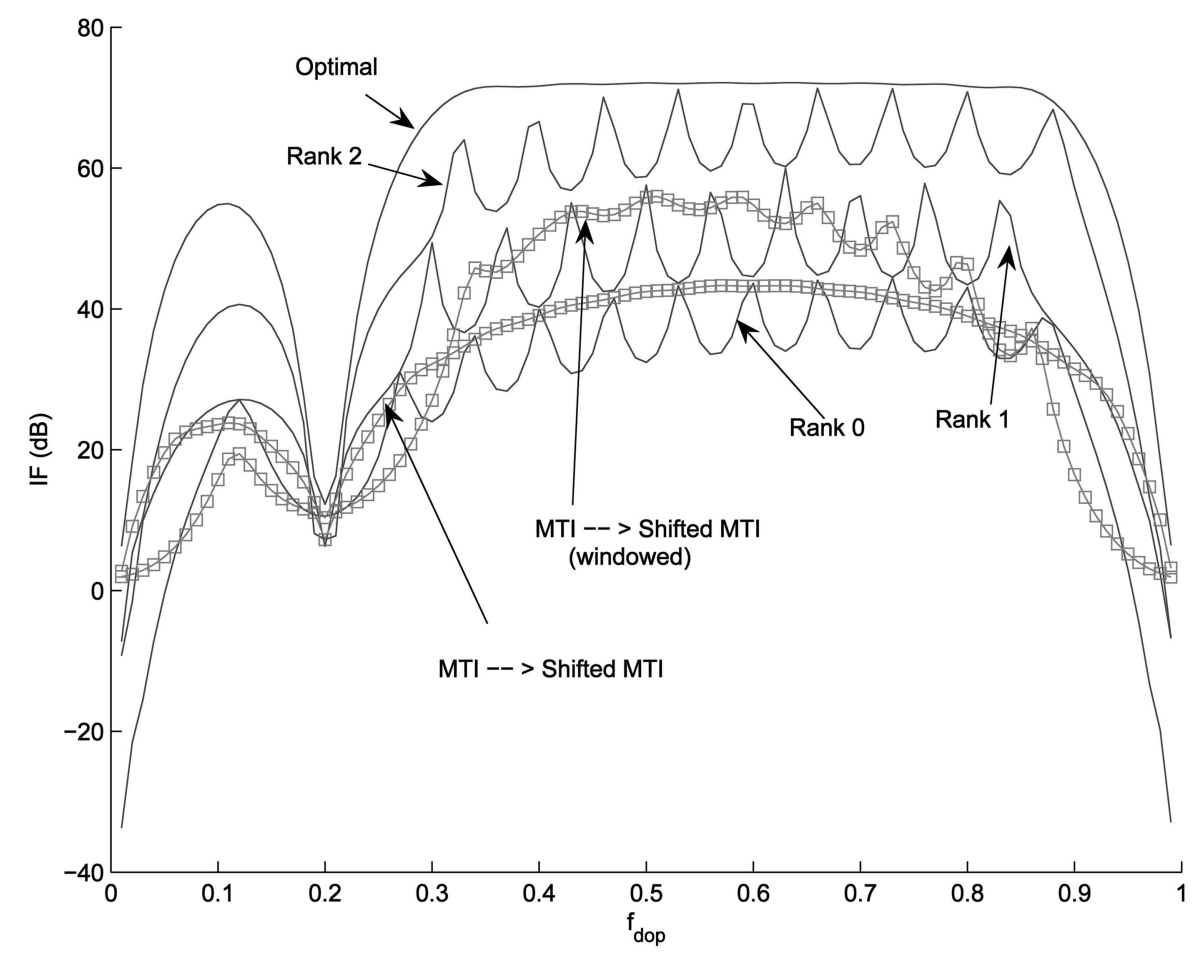

Fig. 4. $N=16, E_{l} / N_{o}=60 \mathrm{~dB}, E_{w} / N_{o}=50 \mathrm{~dB}, f_{w}=0.2 \mathrm{PRF}$.

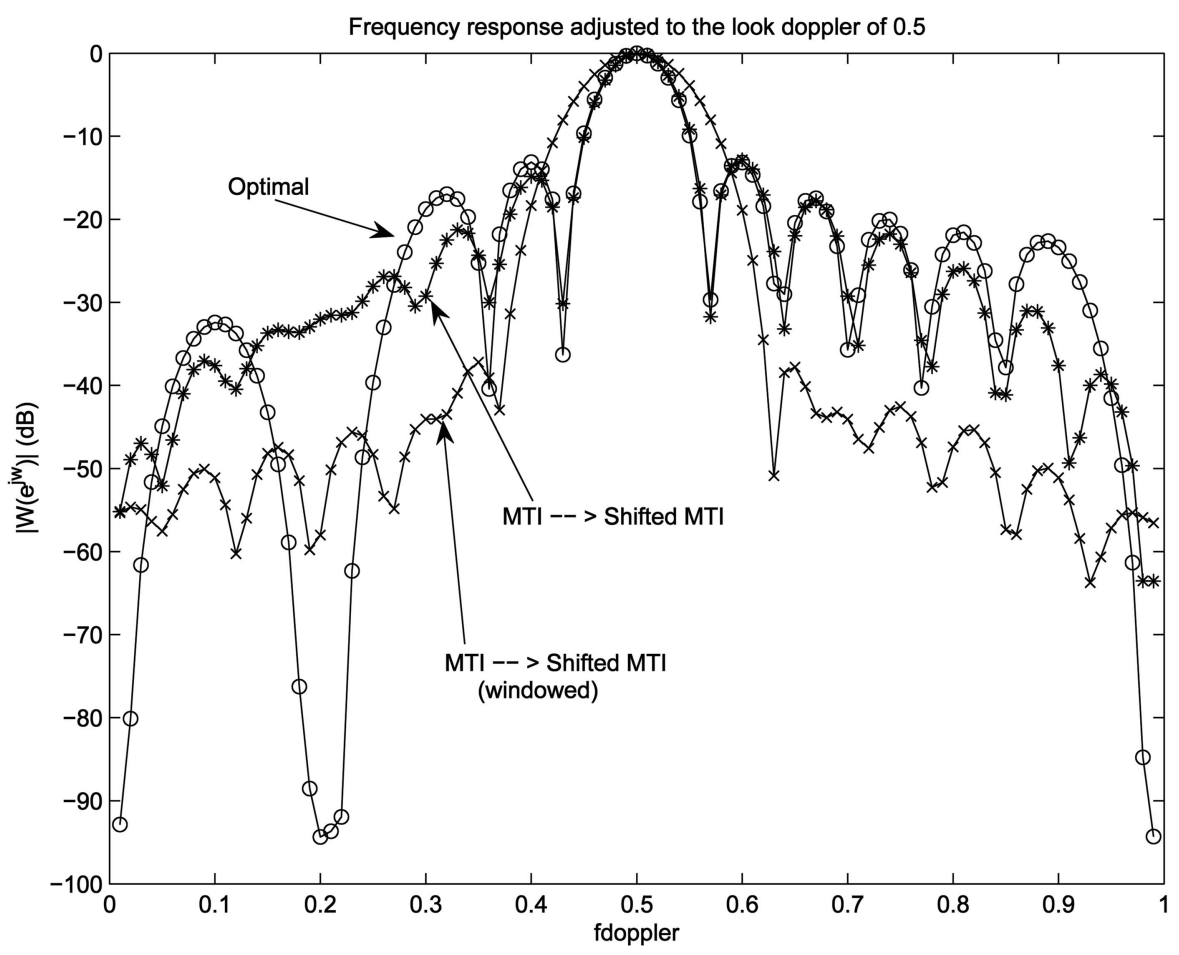

Fig. 5. Frequency response filter of optimal filter and MTI-based filters for look Doppler of 0.5 PRF. Optimal filter is derived under conditions of $N=16, E_{l} / N_{o}=60 \mathrm{~dB}, E_{w} / N_{o}=50 \mathrm{~dB}, f_{w}=0.2 \mathrm{PRF}$.

this paper allow us to implement the optimal solution within a suitable storage-computation trade-off.

\section{CONCLUSIONS}

We have presented methods to cancel coexisting land and weather clutter signals. The SINR optimal method for the implementation of clutter cancellation is known, but it is not practical to implement in many applications. In this paper, we have employed low rank approximations to the weather clutter correlation matrix to define Doppler processors which are more suitable for implementation. The improvement by the proposed approximations in 
comparison with MTI-based methods has been shown to be significant. The method proposed in this paper has been built on the assumption that weather clutter system has the exact same autocorrelation function with the land clutter system except for a shift in the center frequency of its power spectrum due to the radial motion of the weather system. This assumption has led us to design approximate filters whose storage-computation requirements can be selected depending on the capabilities or limitations of the radar platform.

\section{APPENDIX}

We present the matrix inversion formulas for matrices $\left(\mathbf{A}+\beta_{1} \mathbf{e}_{1} \mathbf{e}_{1}^{\mathrm{H}}\right)$ and $\left(\mathbf{A}+\beta_{1} \mathbf{e}_{1} \mathbf{e}_{1}^{\mathrm{H}}+\beta_{2} \mathbf{e}_{2} \mathbf{e}_{2}^{\mathrm{H}}\right)$. The derivation is based on the following result known as the matrix inversion lemma:

$$
(\mathbf{A}+\mathbf{U C V})^{-1}=\mathbf{A}^{-1}-\mathbf{A}^{-1} \mathbf{U}\left(\mathbf{C}^{-1}+\mathbf{V A}^{-1} \mathbf{U}\right)^{-1} \mathbf{V A}^{-1} \text {. }
$$

Inverse of $\left(\mathbf{A}+\beta_{1} \mathbf{e}_{1} \mathbf{e}_{1}^{\mathrm{H}}\right)$ : The inverse of the matrix can be immediately calculated using the matrix inversion lemma when the parameters are set as follows: $\mathbf{C}=\beta_{1}, \mathbf{U}=\mathbf{e}_{1}$, and $\mathbf{V}=\mathbf{e}_{1}^{\mathrm{H}}$. The resultant inverse matrix is

$$
\left(\mathbf{A}+\beta_{1} \mathbf{e}_{1} \mathbf{e}_{1}^{\mathrm{H}}\right)^{-1}=\mathbf{A}^{-1}-\frac{\mathbf{c c}^{\mathrm{H}}}{\sigma_{c}^{2}}
$$

where $\mathbf{c}=\mathbf{A}^{-1} \mathbf{e}_{1}$ and $\sigma_{c}^{2}=1 / \beta_{1}+\mathbf{e}_{1}^{\mathrm{H}} \mathbf{A}^{-1} \mathbf{e}_{1}$.

Inverse of $\left(\mathbf{A}+\beta_{1} \mathbf{e}_{1} \mathbf{e}_{1}^{\mathrm{H}}+\beta_{2} \mathbf{e}_{2} \mathbf{e}_{2}^{\mathrm{H}}\right)$ : The matrix inverse can be calculated using the result given in (19). Calling $\mathbf{B}=\mathbf{A}+\beta_{1} \mathbf{e}_{1} \mathbf{e}_{1}^{\mathrm{H}}$, then we have $\left(\mathbf{A}+\beta_{1} \mathbf{e}_{1} \mathbf{e}_{1}^{\mathrm{H}}+\beta_{2} \mathbf{e}_{2} \mathbf{e}_{2}^{\mathrm{H}}\right)^{-1}=\left(\mathbf{B}+\beta_{2} \mathbf{e}_{2} \mathbf{e}_{2}^{\mathrm{H}}\right)^{-1}$ where the result of (19) can be applied on the right-hand side of the equality. When the inverse of $\mathbf{B}$ is calculated using (19), we get the final result as follows:

$$
\begin{aligned}
(\mathbf{A}+ & \left.\beta_{1} \mathbf{e}_{1} \mathbf{e}_{1}^{\mathrm{H}}+\beta_{2} \mathbf{e}_{2} \mathbf{e}_{2}^{\mathrm{H}}\right)^{-1} \\
= & \mathbf{A}^{-1}-\frac{\mathbf{c} \mathbf{c}^{\mathrm{H}}}{\sigma_{c}^{2}}-\frac{\mathbf{d d ^ { \mathrm { H } }}}{\sigma_{d}^{2}}+2 \operatorname{Re}(\gamma) \frac{\mathbf{d c}^{\mathrm{H}}+\mathbf{c} \mathbf{d}^{\mathrm{H}}}{\sigma_{c}^{2} \sigma_{d}^{2}} \\
& -|\gamma|^{2} \frac{\mathbf{c} \mathbf{c}^{\mathrm{H}}}{\sigma_{c}^{4} \sigma_{d}^{2}}
\end{aligned}
$$

where $\mathbf{c}=\mathbf{A}^{-1} \mathbf{e}_{1}, \mathbf{d}=\mathbf{A}^{-1} \mathbf{e}_{2}, \gamma=\mathbf{e}_{2}^{\mathrm{H}} \mathbf{A}^{-1} \mathbf{e}_{1}, \sigma_{c}^{2}=$ $1 / \beta_{1}+\mathbf{e}_{1}^{\mathrm{H}} \mathbf{A}^{-1} \mathbf{e}_{1}, \sigma_{d}^{2}=1 / \beta_{2}+\mathbf{e}_{2}^{\mathrm{H}} \mathbf{A}^{-1} \mathbf{e}_{2}-|\gamma|^{2} / \sigma_{c}^{2}$.

\section{ACKNOWLEDGMENT}

We would like to thank the members of the Radar Signal Processing Group: Professor F. Canatan, Professor S.: Koç, Professor M. Severçan, Professor Y. Tanik, Dr. Ü. Ç. Doyuran, and B. B. Öztan for lively discussions and many suggestions.

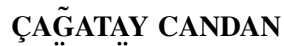

A. ÖZGÜR YILMAZ

Department of Electrical Engineering

Middle East Technical University

Ankara, Turkey

E-mail: (ccandan@metu.edu.tr)

\section{REFERENCES}

[1] Hsiao, J.

On the optimization of MTI clutter rejection.

IEEE Transactions on Aerospace and Electronic Systems, AES-20 (1974), 622-629.

[2] Liu, B.

Clutter suppression using recursive and nonrecursive MTI filters.

IEEE Transactions on Aerospace and Electronic Systems, 24 (May 1988), 210-217.

[3] Schleher, D.

MTI and Pulsed Doppler Radar.

Norwood, MA: Artech House, 1991.

[4] Richards, M.

Fundamentals of Radar Signal Processing.

New York: McGraw-Hill, 2005.

[5] Skolnik, M.

Introduction to Radar Systems.

New York: McGraw-Hill, 2001.

[6] Haykin, S.

Radar signal processing.

IEEE ASSP Magazine, 2 (Apr. 1985), 2-18.

[7] Farina, A., and Protopapa, A.

New results on linear prediction for clutter cancellation. IEEE Transactions on Aerospace and Electronic Systems, 24 (May 1988), 275-286.

[8] Haykin, S., Stehwien, W., Deng, C., Weber, P., and

Mann, R.

Classification of radar clutter in an air traffic control environment.

Proceedings of the IEEE, 79 (June 1991), 742-772.

[9] Doviak, R. J., and Zrnic, D. S.

Doppler Radar and Weather Observations.

New York: Dover Publications, 2006.

[10] Short, R. D.

An Adaptive MTI for weather clutter suppression.

IEEE Transactions on Aerospace and Electronic Systems, 18, 5 (1982), 552-562.

[11] D'Addio, E., Farina, A., and Studer, F.

Performance comparison of optimum and conventional MTI and Doppler processors.

IEEE Transactions on Aerospace and Electronic Systems, 20 (1984), 707-714.

[12] Karp, D., and Anderson, R. J.

Moving target detector (MTD-II).

MIT Lincoln Laboratory, Report ATC-95, 1981.

[13] Stone, M. L., and Anderson, J.

Advances in primary-radar technology.

Lincoln Laboratory Journal, 2, 3 (1989), 363-380.

[14] Cole, E. L., Martineau, M. J., Baker, R. S., and Buswell, S. M.

ASR-12: A next generation solid state air traffic control radar.

Presented at the IEEE Radar Conference 1998. 
D'Addio, E., and Galati, G.

Adaptivity and design criteria of a latest-generation MTD processor.

IEE Proceedings of Radar, Sonar Navigation, 132, 1

(1985), 58-65.

[16] Kumar, P. K., and Prabhu, K. M. M.

Simulation studies of moving-target detection: A new approach with Wigner-Ville distribution.

IEE Proceedings of Radar, Sonar Navigation, 144, 5 (1997), 259-265.

[17] Zhao, X., and Tao, R.

The new algorithm for passive radar MTD based on the fractional correlation.

In Proceedings of the International Conference on Signal Processing, vol. 3, 2004, 2061-2065.

[18] Grajal, J. Quintas, A. G., and Lopez-Risueno, G. MTD detector using convolutional neural networks. In Proceedings of the IEEE Radar Conference, 2005, 827-831.

[19] Muehe, C. E.

The moving target detector.

IEEE Transactions on Aerospace and Electronic Systems, 42, 3 (2006), 1177-1181.

[20] Guerci, J.

Space-Time Adaptive Processing for Radar.

Norwood, MA: Artech House, 2003.

[21] Kay, S. M.

Fundamentals of Statistical Processing, Vol. I: Estimation Theory.

Upper Saddle River, NJ: Prentice-Hall, 1993.

[22] Lancaster, P., and Tismenetsky, M.

The Theory of Matrices.

Burlington, MA: Academic Press, 1985.

[23] Short, R. D.

Adaptive MTI clutter tracker-canceller method and apparatus.

United States Patent 4394658, June 1983.

[24] Mahapatra, P. R., and Zrnic, D. S.

Practical algorithms for mean velocity estimation in pulse Doppler weather radars using a small number of samples. IEEE Transactions on Geoscience and Remote Sensing, GE-21 (Oct. 1983), 491-501.

[25] Gini, F., and Farina, A.

Vector subspace detection in compound-Gaussian clutter. Part I: Survey and new results.

IEEE Transactions on Aerospace and Electronic Systems, 38 (Oct. 2002), 1295-1311.

[26] Candan, C., and Yilmaz, A.

Efficient methods of Doppler processing for coexisting land and weather clutter.

Presented at the IEEE Radar Conference, 2008.
Orthogonal Discrete Frequency-Coding Waveform

Set Design with Minimized Autocorrelation Sidelobes

The autocorrelation sidelobe peak (ASP) levels of discrete frequency-coding waveform with fixed frequency pulses (DFCW-FF) is very large, which is about 0.21 or $-13.2 \mathrm{~dB}$. Replacing the fixed frequency pulses with linear frequency modulated (LFM) pulses can lower the ASP. We investigate the autocorrelation function (ACF) and cross-correlation function (CCF) for DFCW with arbitrary frequency firing order. It is shown that DFCW-LFM has different ACF expression from the DFCW-FF, and the CCF of DFCW-LFM changes distinctly with different frequency firing order. By setting the relationships between the frequency step $\Delta f$, LFM bandwidth $B$ and subpulse duration $T$, we can reduce the ASP, as well as nullify the grating lobes. Then, a modified genetic algorithm (GA) is proposed to numerically search optimal frequency firing order for DFCW-LFM. Some of the designed results are presented. Their correlation properties are effectively improved. Both simulation results and comparisons show that the method of mitigating ASP and the proposed search algorithm are effective for the design of DFCWs with superior aperiodic correlation.

\section{INTRODUCTION}

The successful design of orthogonal code sets with low autocorrelation sidelobe peaks (ASP) and cross-correlation peaks (CP) is crucial for implementing some multiple-input multiple-output (MIMO) radar systems and netted radar systems $[1,2]$. The discrete frequency-coding waveform (DFCW) has been widely used as wideband radar signal to allow high range resolution and improve detection capability [3]. Deng employed a simulated annealing (SA) algorithm to design DFCW with fixed frequency pulses (DFCW-FF) and presented some designed results [4]. In [5], [6], the autocorrelation function (ACF) and cross-correlation function (CCF) of DFCW-FF are investigated. Unfortunately, it shows that ASP for DFCW-FF is comparatively large, which is about 0.21 and has nothing to do with the frequency firing order of DFCW-FF. Here we show that ASP can be further improved. Replacing the

Manuscript received December 5, 2007; revised June 9 and October 15, 2008; released for publication November 19, 2008.

IEEE Log No. T-AES/45/4/935118.

Refereeing of this contribution was handled by M. Rangaswamy.

0018-9251/09/\$26.00 (c) 2009 IEEE 